\title{
Uso de harina de follaje de Tithonia diversifolia en la alimentación de pollos de engorde
}

\author{
Use of Tithonia diversifolia foliage meal in broiler feed \\ Uso de farelo de folhagem de Tithonia diversifolia na ração \\ de frangos de corte
}

\author{
Litsy L Gutiérrez-Castro ${ }^{1 *}$, Víctor L Hurtado-Nery ${ }^{2 *}$ \\ 1 MVZ, MSc. Programa de Maestría Sistemas Sostenibles de Salud Producción Animal Tropical, Facultad de Ciencias \\ Agropecuarias y Recursos Naturales, Universidad de los Ilanos, Villavicencio, Colombia. \\ 2 MVZ, MSc, PhD. Programa de Medicina Veterinaria y Zootecnia, Facultad Ciencias Agropecuarias y Recursos Naturales, \\ Universidad de los Llanos, Villavicencio, Colombia. \\ * Grupo de Estudios en Nutrición Animal, GenA \\ Email: litsy.gutierrez@unillanos.edu.co
}

Recibido: 30 de noviembre de $2018 \quad$ Aceptado: 13 de mayo de 2019

\begin{abstract}
Resumen
El objetivo de este estudio fue evaluar el efecto de la harina de follaje de Tithonia diversifolia como sustitución de fuente tradicional de proteína en dietas para pollos de engorde. Se emplearon 240 pollitos de engorde mixtos de la línea Cobb distribuidos en diseño experimental completamente al azar con arreglo factorial 2 x 4, dos edades de corte 45 y 60 días, con cuatro niveles de inclusión de harina de botón de oro $0,5,10$ y $15 \%$, tres repeticiones y 10 aves por unidad experimental. No hubo efecto de los niveles de Tithonia sobre el consumo de alimento $(P>0,05)$, los niveles de Tithonia influenciaron $(p<0,05)$ la ganancia de peso y la conversión alimentcia. Los resultados obtenidos evidencian que el uso de harina de follaje de Tithonia se puede constituir como una alternativa en la alimentación del pollo de engorde en inclusiones $\leq$ $10 \%$ en la dieta para los dos estados de desarrollo y permite reemplazar parcialmente una materia prima costosa en etapa de finalización.
\end{abstract}

Palabras clave: Tithonia; harina de follaje; alimentación animal

\begin{abstract}
The objective of this study was to evaluate the effect of flour from foliage of Tithonia diversifolia as substitution of traditional source of protein in diets for broilers. We used 240 mixed fattening chicks of the Cobb distributed in a completely randomized experimental design with a $2 \times 4$ factorial arrangement, two cutting ages of 45 and 60 days, with four inclusion levels of $0,5,10$ and $15 \%$ gold button flour, three repetitions and 10 birds per unit experimental. There was no effect of


Tithonia levels on feed intake $(P>0,05)$, Tithonia levels influenced $(p<0,05)$ weight gain and feed conversion. The results obtained in final weight show that the use of gold button foliage flour can be constituted as an alternative in the feeding of the broiler in inclusions $\leq 10 \%$ in the diet for the two stages of development and allows partially replacing a expensive raw material in the final stage.

Key words: Tithonia; foliage; animal feeding

\section{Resumo}

O objetivo desta pesquisa foi avaliar o efeito do farelo de folhagem Tithoni diversifolia em substituicao da fonte tradicional de proteína na dieta para frangos de corte. Foram utilizados 240 pintos de corte da linha Cobb distribuídos em delineamento experimental inteiramente ao acaso em arranjo fatorial $2 \times 4$, duas idades de corte 45 e 60 dias, com quatro níveis de inclusão de 0, 5, 10 e 15\% de farelo de tithonia, três repetições e 10 aves por unidade experimental. Não houve efeito dos níveis de Tithonia sobre o consumo de alimento $(P>0,05)$, os níveis de Tithonia influenciaram $(P<0,05)$ o ganho de peso e a conversão alimentar. Os resultados obtidos sugerem que o uso de farelo de folhagem de Tithonia Os resultados obtidos no peso final evidenciam que o uso de farinha folhagem pode-se constituir como uma alternativa na alimentação de frango em inclusões $\leq 10 \%$ na dieta para as duas fases de desenvolvimento e permite substituir parcialmente matériaprima cara na fase final.

Palavras chave: Tithonia; farelo de follagem; alimentação animal

\section{Introducción}

El aumento continuo de la población mundial, el desarrollo económico global y el incremento del ingreso per cápita de los países en desarrollo, han llevado al incremento en la demanda de alimentos, principalmente en aquellos que son de origen animal. Este fenómeno mundial, conlleva a que la producción pecuaria de América Latina y el Caribe enfrente actualmente un desarrollo acelerado de productos agropecuarios, que a pesar de llegar a presentar mejor productividad y uniformidad en el producto final, requiere altos costos de inversión y dependencia del mercado internacional de cereales; además son alarmantes las cifras que indican la degradación de los recursos naturales, vulnerabilidad al cambio climático y niveles de pobreza en las zonas rurales (FAO, 2014).

El aumento en la producción de especies menores constituye una estrategia para suplir las necesidades de proteína de origen animal que demandan los hogares; este hecho conlleva a que los productores aumenten la cantidad de materia prima que se destina para la alimentación animal y de esta manera surge la necesidad de promover opciones para disminuir la dependencia de los alimentos concentrados y mantener la producción de proteína animal. El interés por los forrajes arbóreos para alimentación animal ha sido estudiado permanentemente en los últimos años, llevando a que la agroforesteria sea una alternativa para el desarrollo de sistemas agropecuarios sostenibles, con ventajas en cobertura vegetal, protección y mejoramiento del suelo, recuperación y conservación de fuentes de agua, y fuente de alimento para animales y el hombre (Sarria et al., 2005). El uso de forrajes en Colombia está orientado básicamente a gramíneas y leguminosas, sin embargo, en los últimos años se han evaluado otras especies vegetales que se encuentran relegadas como cultivos nativos o malezas, sin ningún tipo de beneficio pero que pueden tener potencial de uso en alimentación animal logrando establecer un complemento nutricional para las diferentes producciones agropecuarias.

Según González (2014) el uso de plantas forrajeras en la dieta de diferentes especies animales es una de las opciones para la producción eficiente y rentable en sistemas agropecuarios. Ante estas condicionantes, se buscan fuentes proteicas más económicas, evaluando específicamente follajes de algunas especies arbóreas que, por su potencialidad, pueden garantizar dietas adecuadas nutricionalmente para animales de producción en clima cálido, como en los Llanos Orientales. Sarria (1999) destaca el rango de adaptación que tiene la Tithonia diversifolia que se observa en muy buen estado y producción desde el nivel del mar hasta los 2400 msnm, en suelos pobres y de mediana fertilidad, su contenido de humedad alrededor del $80 \%$ y contenido alto de proteína de $20 \%$, asimismo infiere que comparada con la trichanthera gigantea presenta $31 \%$ más de proteína bruta, $26 \%$ más de proteína verdadera, $6 \%$ más proteína de naturaleza aminoacidica, 22\% menos de fibra insoluble y $60 \%$ menos de nitrógeno ligado a la fibra insoluble, demostrando potencial del forraje.

El botón de oro (Tithonia diversifolia), se puede cultivar y utilizar como componente nutricional (Ríos, 2002; Mahecha y Rosales 2005; Gonzales et al., 2014); pero que aún no es aprovechada completamente como opción para la suplementación nutricional por productores de especies menores o la usan empíricamente en 
los animales sin conocer el valor nutricional. Sanabria y Ávila (2015), reportan que posee un nivel de proteína que varía de 14,8 a 28,5\% de proteína y aceptable digestibilidad de nutrientes (Nieves et al., 2011) en especies como conejos, lo que permite evidenciar su potencialidad nutritivo, estos aspectos brindan la posibilidad de uso como materia prima en dietas para pollos de engorde.

El consumo nacional de productos avícolas, especialmente de la carne de pollo, ha experimentado un constante crecimiento que se evidencia con el incremento en el consumo per cápita (FENAVI, 2018), e infieren que el crecimiento de la producción avícola en el país ha sido sostenido y permanente en los últimos cinco años; este crecimiento de la industria avícola ejerce un profundo efecto en la demanda de alimentos animales y materias primas. La nutrición en aves de corral está orientada a la elección de dietas que cubran los requerimientos nutricionales del animal y además que estén en función de su disponibilidad y precio, para obtener aves sanas y productivas. Por lo anterior, el objetivo de este estudio fue evaluar el efecto de la harina de follaje de botón de oro (Tithonia diversifolia) como sustitución de fuente tradicional de proteína en dietas para pollos de engorde en niveles de 0, 5, 10 y $15 \%$ de la dieta, teniendo como variables de respuesta la ganancia de peso, consumo de alimento, conversión alimenticia.

\section{Metodología}

\section{Localización}

El estudio se llevó a cabo durante los meses de enero y febrero de 2018 en las instalaciones de la Granja Barcelona en la unidad de pollos, del Departamento de Producción de la Facultad de Ciencias Agropecuarias y Recursos Naturales de la Universidad de los Llanos, ubicada en el km 12 vía Puerto López, municipio de Villavicencio, Meta. Las características fisiográficas de la región son altitud de 467 metros sobre el nivel del mar, temperatura promedio de 27 grados centígrados, precipitación promedio anual entre 1830 a $3568 \mathrm{~mm}$ y humedad relativa del $85 \%$.

\section{Trabajo Experimental}

En un cultivo de Tithonia establecido en la granja Barcelona de la Universidad de los Llanos, se realizó la poda de las plantas con el objetivo de homogeneizar el cultivo para proceder a realizar la recolección del follaje de Tithonia o Botón de Oro, el material vegetal se recolectó en época de verano, en dos edades de corte 45 y 60 días. El material recolectado, fue deshidratado en secador solar natural de techo plástico, en placa de cemento con área de $20 \mathrm{~m}^{2}$, durante 3 días, luego fue empacado en papel periódico y llevado a horno por 24 horas a $60^{\circ} \mathrm{C}$ para un secado homogéneo, y finalmente procesado y transformado en harina en molino Desintegrador Pinheiro Pd5. La harina de Tithonia

Tabla 1. Composición centesimal de la dieta balanceada y dieta con inclusión de botón

\begin{tabular}{|l|c|c|c|c|}
\hline \multirow{2}{*}{ Ingredientes } & \multicolumn{4}{c|}{ Cantidad en 100 kg de alimento } \\
\cline { 2 - 5 } & BO 0\% & C BO 5\% & C BO 10\% & C BO 15\% \\
\hline Harina de Maíz amarillo & 52,0 & 52 & 52 & 52 \\
Harina de soya & 23,3 & 21,5 & 19,6 & 17 \\
Harina de arroz & 7,0 & 7,0 & 7,0 & 7,0 \\
Mogolla de trigo & 5,0 & 5,0 & 5,0 & 5,0 \\
Torta de Palmiste & 8,0 & 8,0 & 8,0 & 8,0 \\
Carbonato de calcio & 1,0 & 1,0 & 1,0 & 1,0 \\
Fosfato bicálcico & 1,1 & 1,1 & 1,1 & 1,1 \\
Aceite vegetal & 0,5 & 0,5 & 0,5 & 0,5 \\
Premezcla vitaminas-minerales & 0,90 & 0,90 & 0,90 & 0,90 \\
Sal común & 0,40 & 0,40 & 0,40 & 0,40 \\
Treonina & 0,10 & 0,10 & 0,10 & 0,10 \\
Metionina & 0,12 & 0,12 & 0,12 & 0,12 \\
Lisina & 0,24 & 0,24 & 0,24 & 0,24 \\
Inerte & 0,79 & 0,3 & 0,3 & 0,3 \\
Harina de Tithonia & 0,0 & 1,84 & 3,8 & 6,4 \\
\hline
\end{tabular}

* La sustitución del \% de harina de botón de oro se realizó sobre la fuente de proteína tradicional (harina de soya) 
fue homogenizada con la ración basal, constituida por maíz, torta de soya, harina de arroz, mogolla de trigo, torta de palmiste, fosfato bi-cálcico, carbonato de calcio, vitaminas, sal y aminoácidos sintéticos (Tabla 1), se prepararon en la granja Barcelona, para atender a los requerimientos nutricionales (tabla 2) sugeridos por Rostagno (2011).

Se utilizaron 240 pollitos de engorde mixtos de la línea Cobb, alimentados con concentrado comercial de preiniciación (proteína 21\%) hasta los 22 días de edad, luego con peso promedio de $800 \mathrm{~g}+/-100 \mathrm{~g}$, fueron alojados en grupos de 10 animales en jaulas de piso en concreto con cama en cascarilla, separadas por módulos con área de $1 \mathrm{~m}^{2}$, provistas de comedero y bebedero cada una.

Se dividieron en tratamiento testigo a base de concentrado comercial, y 7 tratamientos con inclusión de harina de botón de oro $(0,5,10$ y $15 \%)$, con tres repeticiones y 10 aves por unidad experimental. Para un total de 240 aves; que tuvieron periodo de acostumbramiento durante 5 días y se alimentaron en 3 raciones hasta alcanzar los 45 días de edad.

\section{Diseño experimental}

El diseño experimental fue completamente al azar con arreglo factorial $2 \times 4$. Dos edades de corte 45 y 60 días, cuatro niveles de inclusión 0, 5, 10 y 15\% de harina de botón de oro y tres repeticiones de 10 aves cada uno. Las variables evaluadas fueron: consumo de alimento total (CAT), ganancia de peso total (GPT) y conversión alimenticia (CA).

\section{Procesamiento de información}

La información se registró en una base de datos de Excel del programa Microsoft 2010. La comparación estadística de las variables técnicas entre tratamientos se realizó mediante el análisis de varianza (ANOVA), significancia estadística del $5 \%$, y el contraste a posteriori de medias (prueba de Tukey) utilizando el software estadístico SPSS Statistics 20.0.

\section{Resultados}

En las tabla 2 se presenta los datos finales de los tratamientos, siendo confrontados de manera simultánea en condiciones de manejo similares. El consumo de alimento no presentó diferencias estadísticamente entre tratamientos $(P>0,05)$, el suministro mínimo estimando se realizó de acuerdo a las tablas de consumo (SOLLA, 2017) y es afín con lo reportado por Bucardo y Pérez; (2015) para pollo de engorde (ave/día) durante la fase productiva de engorde, cuyo promedio estuvo en 166+-- 4 g/ave/día, sin presentar diferencias estadísticas significativas $(P>0,05)$ entre los tratamientos. En relación al consumo de alimento total no se encontraron diferencias estadísticas $(P>0,05)$ entre los tratamientos, sin embargo para el tratamiento Dieta + Botón 60-15\% se presentó el menor consumo de alimento total vs los demás tratamientos.

Los tratamientos que registraron la mejor ganancia de peso total $(\mathrm{P}<0,05)$ fueron Testigo, Dieta $+0 \%$ Botón y Dieta + Botón $45-5 \%$ (1.26, 1.16 y 1.16 respectivamente (Tabla 3). Estos resultados se pueden atribuir a que la dieta balanceada y con botón de oro a los 45 días, presentaron contenido adecuado de energía y proteína, similares al concentrado comercial.

Tabla 2. Composición bromatológica (proximal) de 3 de las dietas evaluadas

\begin{tabular}{|c|c|c|c|c|c|c|}
\hline Parámetro & $\begin{array}{c}\text { Dieta } \\
\text { balanceada }\end{array}$ & $\begin{array}{c}\text { Dieta+ } \\
\text { 10\%Tithonia } 45\end{array}$ & $\begin{array}{c}\text { Dieta+ } \\
\text { 10\%Tithonia } 60\end{array}$ & $\% \mathrm{CV}$ & EEM & $\mathbf{p}$ \\
\hline Materia Seca, \% & 90,26 & 91,99 & 90,45 & 0,54 & 0,2818 & $<0,010$ \\
\hline Ceniza, \% & 19,08 & 21,11 & 18,55 & 3,27 & 0,5058 & $<0,010$ \\
\hline Materia Orgánica, \% & 71,10 & 70,80 & 71,45 & 1,03 & 0,4221 & NS \\
\hline Nitrógeno Total, \% & 3,84 & 3,84 & 3,86 & 3,62 & 0,0509 & $<0,050$ \\
\hline Proteína Cruda, \% & 24,13 & 24,02 & 24,12 & 22,62 & 0,3186 & $<0,050$ \\
\hline Extracto Etéreo, \% & 1,92 & 2,85 & 2,52 & 12,86 & 0,1805 & $<0,050$ \\
\hline Fibra cruda, \% & 38,78 & 41,95 & 45,94 & 1,93 & 0,4706 & $<0,010$ \\
\hline Energía Metabolizable (EM) & 3,05 & 2,84 & 2,54 & 1,31 & 0,0213 & $<0,010$ \\
\hline
\end{tabular}

Valores expresados en porcentaje, con excepción de EM (Mcal/Kg MS).EEM= Error estándar de la media, CV= Coeficiente de Variación, p= Significancia estadística, NS: $\mathrm{P}>0,050$. 
Tabla 3. Resultados productivos de pollos alimentados con diferentes niveles de harina de follaje de Tithonia

\begin{tabular}{|l|l|l|l|}
\hline \multicolumn{1}{|c|}{ Tratamiento } & \multicolumn{1}{|c|}{ CAT $(\mathbf{K g})$} & \multicolumn{1}{c|}{ GTP $(\mathbf{K g})$} & \multicolumn{1}{c|}{ CA } \\
\hline 1. Concentrado comercial & $2,5512+/-0,03$ & $1,2697+/-0,02^{\mathrm{a}}$ & $2,0+/-0,57^{\mathrm{a}}$ \\
\hline 2. Dieta 0\% Botón de oro & $2,5292+/-0,09$ & $1,1609+/-0,06^{\mathrm{a}}$ & $2,2+/-0,57^{\mathrm{b}}$ \\
\hline 3. Dieta + Botón 45- 5\% & $2,5390+/-0,06$ & $1,1675+/-0,08^{\mathrm{a}}$ & $2,2+/-0,2{ }^{\mathrm{b}}$ \\
\hline 4. Dieta +Botón 45- 10\% & $2,5487+/-0,03$ & $1,1230+/-0,04^{\mathrm{b}}$ & $2,3+/-0,57^{\mathrm{b}}$ \\
\hline 5. Dieta +Botón 45- 15\% & $2,5571+/-0,04$ & $1,0794+/-0,04^{\mathrm{b}}$ & $2,3+/-0,57^{\mathrm{b}}$ \\
\hline 6. Dieta +Botón 60- 5\% & $2,5270+/-0,03$ & $1,1207+/-0,05^{\mathrm{b}}$ & $2,3+/-0,57^{\mathrm{b}}$ \\
\hline 7. Dieta +Botón 60- 10\% & $2,5542+/-0,04$ & $1,1504+/-0,08^{\mathrm{a}}$ & $2,2+/-0,1^{\mathrm{b}}$ \\
\hline 8. Dieta +Botón 60- 15\% & $2,4853+/-0,1$ & $1,0681+/-0,1^{\mathrm{b}}$ & $2,3+/-0,23$ b \\
\hline P & 0,227 & 0,088 & 0,053 \\
\hline \%CV & 2,43 & 6,49 & 5,39 \\
\hline
\end{tabular}

Valores con letra diferente indican diferencias estadísticas significativas con prueba de Duncan, $(\mathrm{P}<0.05)$. CAT: Consumo Alimento total por ave, GTP: Ganancia total de peso, CA: Conversión alimenticia.

El efecto de los tratamientos sobre la conversión y la ganancia de peso de las aves fue significativa, arrojando valores bajos para el tratamiento 1 o testigo con respecto a los demás tratamientos en la variable de CA y con respecto al GTP el tratamiento 1 y 2 obtuvieron los valores más altos con respecto a los tratamientos con inclusión de harina de Tithonia (tabla 3). A su vez, el peso corporal de las aves en los tratamientos T1, T2, T3 y T7 presentó valor significativamente $(\mathrm{P}<0,05)$ más alto al de los tratamientos T4, T5, T6 y T8, e inclusive el T5 y T8 obtuvieron los valores más bajos.

La prueba de promedios evidencia al tratamiento testigo como el de mejor comportamiento productivo comparado con los demás tratamientos, siendo el tratamiento con $0 \%$ de inclusión de botón de oro con (2.2) como el más bajo, seguido con valores similares para las dietas con adición de $5 \%$ de harina a los 45 días y $10 \%$ con harina de 60 días, para los tratamiento con adición de 15\% para amabas edades de corte la conversión alimenticia fue relativamente alta como se puede apreciar en la tabla 2, expresando ser menos eficientes para la alimentación de las aves.

\section{Discusión}

Los resultados de CAT coinciden con lo reportado por Bucardo y Pérez (2015) quienes encontraron que el consumo total de alimento fue estadísticamente igual al suministrar en pollos dietas con inclusión de harina de hoja de Moringa oleifera al final del ciclo productivo
(42 d); por otra parte Connolly (2017), quien suministró un alimento a pollo de engorde elaborado que contenía $10 \%$ de harina de raíz de yuca y $10 \%$ de harina de forraje de yuca, encontró diferencias $(P<0,05)$ en el consumo total. Asimismo, los resultados obtenidos para consumo de alimento concuerdan con lo informado por Valdivié et al., (2008), quien sustituyó de forma total al maíz por harina de yuca en dietas para pollos de engorde e indica que no hay diferencias en palatabilidad y aceptación por parte de las aves, cuando se ofertan, en forma de harina.

El contenido de fibra de los tratamientos es más alto que en el reportado para el concentrado comercial, lo cual pudo haber afectado el valor energético y la digestibilidad de las dietas, pero no influyó significativamente $(P>0,05)$ la ganancia de peso de los animales y permitió sustentar valores similares, concordantes con los observados para esta variables cuando se utiliza dietas convencionales. Asimismo, concuerdan con lo expresado por Casamachin et al., (2007) donde los resultados demuestran que no existieron diferencias estadísticas entre el tratamiento testigo y la dieta no convencional con 5, 10 y 15\% de inclusión de harina de morera para la variable ganancia de peso, contrario a los resultados expresados por López et al., (2012) que encontraron mayor ganancia de peso total en finalización 1794,4 g $(\mathrm{P}<0,05)$ al incluir harina de hoja de bore (Alocasia macrorhiza) en proporciones de 5\% en la alimentación de pollos machos de la línea Ross. Trujillo y Escobar (2012) observaron que la inclusión de 
harina de plantas forrajeras tuvo efecto inverso sobre el comportamiento productivo del pollo de engorde, ya que a medida que aumenta el nivel de inclusión en la dieta en 25, 50 y $75 \%$, disminuye la ganancia de peso en $31 \%$, 52\% y $83 \%$, respectivamente.

Meza et al., (2014) argumentan que, a mayor nivel energético en la ración, la ganancia de peso y conversión alimenticia mejoran; y como ya es conocido los broilers responden eficientemente a dietas con altos contenidos de energía, por lo cual el tratamiento testigo presentó los mejores valores para GTP. Por su parte, Valencia et al., (2007), mencionan que la incorporación de fibra deteriora los niveles de energía en la dieta y se pueden usar niveles de fibra de hasta $7-10 \%$ sin efecto negativo en el rendimiento biológico de pollos; lo cual coincide con lo encontrado en este estudio en las aves a las que se les suministró los tratamiento con el 15\% de inclusión de Tithonia o botón de oro, ya que al incrementar el contenido de tithonia diversifolia de 10\% a $15 \%$ en la dieta, las ganancias de peso disminuyeron significativamente $(P<0,05)$; ya que excesos de fibra en la ración pueden causar efectos negativos sobre la productividad en monogástricos jóvenes, relacionado con la palatabilidad, reducción de digestibilidad de los nutrientes y sensación de saciedad, haciendo que el pollo sea incapaz de consumir la cantidad de alimento necesaria, para cubrir sus necesidades energéticas (López et al., 2012). Es el caso de lo encontrado por Girón y Cubides (2018) en un estudio con pollo campesino, alimentados con $75 \%$ de concentrado comercial y 25\% T. Diversifolia + Gliricidia sepium + maíz; y otros con $50 \%$ de concentrado y $50 \%$ T. Diversifolia +Gliricidia sepium+maíz; y que alcanzaron a la séptima semana pesos de: 1.150 y 1.127 g y el índice de CA fue superior a 3.0 para ambos tratamientos.

El índice de conversión es una medida de la productividad del animal y se define como la relación del alimento usado para conseguir el peso final, por lo cual mientras más bajo sea el índice de conversión más eficiente es el alimento (Casamachin et al., 2007). Trujillo y Escobar (2012), encontraron valores similares a los de este estudio, con diferencias estadísticas significativas $(\mathrm{P}<0,05)$ entre los tratamientos para la variable conversión alimenticia, ya que comparado con el testigo se afectó negativamente, la inclusión de 25, 50\% de harina de forrajes Trichanthera gigantea Tithonia diversifolia, Saccharum spp, Alocasia macrorrhiza y Musa paradisiaca (CA 1.62, 2.20, 3.66 respectivamente). Contrario a lo mencionado por Arboleda et al., (1994), citado por Valencia et al., (2007); quien concluye que la eficiencia de pollos en la utilización de nutrientes, mejoró conforme se incrementaron los niveles de fibra en la dieta, la utilización de los nutrientes se refleja en menor consumo de alimento y mejor rendimiento productivo.

Bucardo y Pérez (2015), reportan conversión alimenticia de 1.60 con concentrado comercial; así mismo, Connolly (2017), reporta conversión alimenticia de 2.12 con concentrado comercial y al incluir dietas no convencionales, estos mismos autores encontraron valores de conversión alimenticia de 1.64 y 2.28 sin diferencias significativas. Estos resultados difieren con los constatados en este trabajo ya que la CA de las aves alimentadas con concentrado comercial comparado con los del alimento no convencional presentó diferencias significativas.

Por otro lado, Valencia et al., (2007), reemplazando el concentrado por Trichanthera gigantea en niveles 7.12 y $17 \%$ en la etapa de engorde no encontraron diferencias significativas en las variables evaluadas de ganancia de peso y conversión alimenticia.

Los resultados obtenidos en peso final evidencian que el uso de harina de follaje de botón de oro se puede constituir, como una alternativa en la alimentación del pollo de engorde por lo menos hasta la proporción de $10 \%$ en la dieta para las fases de desarrollo y permite reemplazar parcialmente la fuente de proteína aportada por una materia prima costosa como lo es la harina de soya, siendo mejor las raciones con la harina de 60 días de corte (20.2 de proteína). Este resultado se explica debido a que las aves al no presentar dientes, los alimentos digeridos son descompuestos de forma mecánica y química en el aparato digestivo y el proceso digestivo hace posible la liberación de nutrientes contenidos en los alimentos, así mismo, hace posible la absorción y distribución uniforme de estos nutrientes en el cuerpo del ave (Girón y Cubides, 2018).

Los tratamientos alternativos conservaron la eficiencia en consumo, ganancia de peso y conversión no presentaron diferencias con el tratamiento de alimento balanceado y el testigo, debido probablemente a que el aporte de requerimientos fue suplido satisfactoriamente por las dietas suministradas; igualmente, se evidenció comportamiento heterogéneo entre los tratamientos alternativos presentando mejor eficiencia productiva la dieta con inclusión de harina de botón de oro (Tithonia diversifolia) hasta del 10\% para ambas edades de corte, como también, se recomienda que junto con el material forrajero alternativo probado en este estudio, se compruebe su uso completo (tallo - hojas) sobre el comportamiento productivo de pollos de engorde. 


\section{Agradecimientos}

Los autores expresan sus agradecimientos a la Dirección General de Investigaciones de la Universidad de los Llanos por el apoyo financiero. Al Departamento de Producción Animal de la Facultad de Ciencias Agropecuarias y Recursos Naturales por permitir las instalaciones para el desarrollo de la fase experimental de esta investigación.

\section{Referencias}

Bucardo E, Pérez J. Inclusión de harina de hojas de Marango (Moringa oleífera) en la alimentación de pollos de engorde y su efecto en el comportamiento productivo. Tesis, Universidad Nacional Agraria-UNA, 2015. Disponible en: http://repositorio.una.edu. ni/id/eprint/3243

Casamachin M, Ortiz D, López, Fredy J. Evaluación de tres niveles de inclusión de morera (morus alba) en alimento para pollos de engorde. Facultad de Ciencias Agropecuarias 2007;5(2):64-71. Disponible en: http://revistabiotecnologia.unicauca.edu.co/revista/index.php/biotecnologia/article/viewFile/9/46

COBB. 2012. Guía de Manejo del Pollo de Engorde. cobb-vantress. com. Disponible en: http://www.pronavicola.com/contenido/ manuales/Cobb.pdf

Connolly D. 2017. Inclusión de harina de follaje y raíz de yuca (Manihot esculenta crantz), en la alimentación de pollos de engorde y su efecto en el comportamiento productivo. Tesis, Universidad Nacional Agraria Managua. Disponible en: http://repositorio. una.edu.ni/3500/1/tnl02c752.pdf

FAO: Producción pecuaria en América Latina y el Caribe, 2014. Disponible en: http://www.fao.org/americas/prioridades/produccion-pecuaria/es/

FENAVI: El sector avícola en Colombia creció 4.5\% en 2018. Disponible en: https://fenavi.org/comunicados-de-prensa/el-sectoravicola-crecio-45-en-2018/

Girón D, Cubides Y. 2018. Evaluación de ganancia de peso y conversión alimenticia en pollo campesino bajo manejo de estabulación sustituyendo el 25 y 50\% de la ración comercial por Tithonia Diversifolia, Gliricidia Sepium y Zea mayz. Tesis, Universidad Nacional Abierta y a Distancia, UNAD.

Gónzalez J, Hahn C, Narvaez W. Características botánicas de Tithonia diversifolia (Asterales: Asteraceae) y su uso en la alimentación animal. Bol Cient Mus Hist Nat. U. de Caldas. 2014;18(2):45-58.

López MF; Caicedo GA; Alegría FG. Evaluación de tres dietas con harina de hoja de bore (Alocasia macrorrhiza) en pollos de engorde. Revista MVZ Córdoba, 2012;17(3):3236-3242.

Meza G, Loor N, Sánchez A, Avellaneda J, Meza C, Vera D, Cabanilla $M$, et al. Inclusión de harinas de follajes arbóreos y arbustivos tropicales (Morus alba, Erythrina poeppigiana, Tithonia diversifolia E Hibiscus rosa-sinensis) en la alimentación de cuyes (Cavia porcellus Linnaeus). Rev Fac Med Vet Zoot. 2014;61(3):258-269.

Nieves D, Terán O, Cruz L, Mena M, Gutiérrez F, Ly J. Digestibilidad de nutrientes en follaje de árnica (tithonia diversifolia) en conejos de engorde. Rev Tropical and Subtropical Agroecosystems. 2011;14(1):309-314.

Rostagno S. 2011. Tablas brasileñas para aves y cerdos: composición de alimentos y requerimientos nutricionales. Universidad Federal de Viçosa. 3. ed. - Viçosa, MG: UFV, DZO. P.p 259.

Sanabria E, Avila C, Iveth Y. 2015. Producción de follaje de la especie botón de oro (tithonia diversifolia) utilizando 5 técnicas de siembra con fines de alimentación animal. Tesis, UNAD, $\mathrm{Pp}$ 15. Disponible en: https://repository.unad.edu.co/bitstream/10596/3713/1/86047820.pdf

Sarria P. 1999. Forrajes arbóreos en la alimentación de monogástricos. En: Memorias I Seminario Avances en la Agroforestería Pecuaria en el Departamento de Antioquia, Universidad Nacional de Colombia Sede Medellín. Disponible en: http://bibliotecadigital. agronet.gov.co/bitstream/11348/4019/1/20061127103814_ Forrajes $\% 20$ arbores $\% 20$ alimentacion $\% 20$ monogastricos.pdf

Sarria P, Leterme P, Londoño A, Botero M. 2005. Valor nutricional de algunas forrajeras para la alimentación de monogástricos. Memorias Curso Pre-evento Alimentación no convencional para Monogástricos en el Trópico VIII Encuentro de Nutrición y Producción de Animales Monogástricos. UNELLEZ-Guanare. Disponible en: http://www.avpa.ula.ve/eventos/viii_encuentro_monogastricos/curso_alimentacion_no_convencional/conferencia-10.pdf

Solla S.A. - Excelencia avícola. 2017. Dirección nacional avicultura balanceados. Manual de manejo para pollo de engorde. Disponible en: https://www.solla.com/sites/default/files/productos/ secciones/adjuntos/MANUAL\%20\%20POLLO\%20DE\%20ENGORDE\%20SOLLA\%202017.pdf

Trujillo A, Escobar A. Evaluación de la sustitución de concentrado comercial por harina de forrajeras en pollos de engorde Ross. Agroecología: Ciencia y Tecnología Servicio Nacional de Aprendizaje - Sena. Agroecol Cienc Tecnol. 2012;1(1):6-12. Disponible en: http://revistas.sena.edu.co/index.php/agroeccyt/article/view/842/932

Valdivié M, Leyva C, Cobo R, Ortiz A, Dieppa O, Febles M. Sustitución total del maíz por harina de yuca (Manihot esculenta) en las dietas para pollos de engorde Revista Cubana de Ciencia Agrícola. 2008;42(1):61-64. Disponible en: http://www.redalyc.org/articulo.oa?id=193015413010

Valencia J, Sarria E, Rivera D. 2007. Efecto de tres niveles de inclusión de nacedero (Trichanthera gigantea) y materias primas convencionales en alimentación de pollos de engorde en el municipio de Popayán, Cauca. Tesis, UNAD, Popayán.

Litsy Gutiérrez: https://orcid.org/0000-0002-7395-5348 Víctor Hurtado: https://orcid.org/0000-0002-9890-5726 